\title{
Enhanced ethanol production and reduced glycerol formation in fps $1 \Delta$ mutants of Saccharomyces cerevisiae engineered for improved redox balancing
}

\author{
Clara Navarrete, Jens Nielsen and Verena Siewers ${ }^{*}$
}

\begin{abstract}
Ethanol is by volume the largest fermentation product. During ethanol production by Saccharomyces cerevisiae about 4-5\% of the carbon source is lost to glycerol production. Different approaches have been proposed for improving the ethanol yield while reducing glycerol production. Here we studied the effect of reducing glycerol export/formation through deletion of the aquaglyceroporin gene FPS1 together with expressing gapN encoding $\mathrm{NADP}^{+}$-dependent non-phosphorylating glyceraldehyde-3-phosphate dehydrogenase from Streptococcus mutans and overexpressing the ATP-NADH kinase gene UTR1 from S. cerevisiae. This strategy will allow reducing the redox balance problem observed when the glycerol pathway is blocked, and hereby improve ethanol production. We found that our strategy enabled increasing the ethanol yield by $4.6 \%$ in the case of the best producing strain, compared to the reference strain, without any major effect on the specific growth rate.
\end{abstract}

Keywords: Saccharomyces cerevisiae; Ethanol production; Glycerol; Redox balancing

\section{Introduction}

Ethanol is both in terms of market value and volume one of the most important products from the biotechnology industry. Even though this process is highly optimized there is still interest to improve the productivity, the robustness of the strains and the product yield (van Maris et al. 2006; Hahn-Hagerdal et al. 2007). There are different parameters that determine the economy of this industrial bioprocess; one of the most important ones is the price of the feedstock (Wyman and Hinman 1990). Therefore, it is of utmost importance to increase the ethanol yield as well as the carbon source utilization. During ethanol production by Saccharomyces cerevisiae, glycerol is a major by-product, representing 4-5\% of the carbon source consumption, in addition to biomass, carbon dioxide and a number of other by-products such as acetic acid, pyruvic acid or succinic acid (Nissen et al. 2000b; Wyman and Hinman 1990; Zhang and Chen 2008; Oura 1977).

\footnotetext{
*Correspondence: siewers@chalmers.se

Department of Chemical and Biological Engineering, Chalmers University of Technology, Kemivägen 10, SE-41296 Göteborg, Sweden
}

During anaerobic fermentation, the respiratory chain is not functional and the NADH generated in connection with cell growth must be re-oxidized to $\mathrm{NAD}^{+}$by formation of glycerol, in order to avoid an imbalance in the $\mathrm{NAD}^{+} / \mathrm{NADH}$ ratio (Nissen et al. 2000a). Furthermore, under osmotic stress conditions, glycerol is produced and accumulated in the cell as an osmolyte, to protect cells against cell lysis (Andre et al. 1991; Larsson et al. 1993; Ansell et al. 1997). Glycerol is synthethized from dihydroxyacetone phosphate in two steps catalysed by Gpd1/Gpd2 (glycerol-3-phosphate dehydrogenases) and Gpp1/Gpp2 (glycerol-3-phosphate phosphatases), respectively (Figure 1). Expression of GPD1 and GPP2 is induced by high osmolarity, whereas expression of GPD2 and GPP1 is stimulated under anaerobic conditions (Larsson et al. 1993; Eriksson et al. 1995; Nissen et al. 2000a). It has been reported that the formation of glycerol could be decreased by the consumption of NADH by alternative metabolic pathways (Vemuri et al. 2007; Bro et al. 2006). It has also been shown that deletion of either the GPD1 or GPD2 gene led to a decrease in the glycerol yield (Guo et al. 2009; Michnick et al. 1997; Nissen et al. 


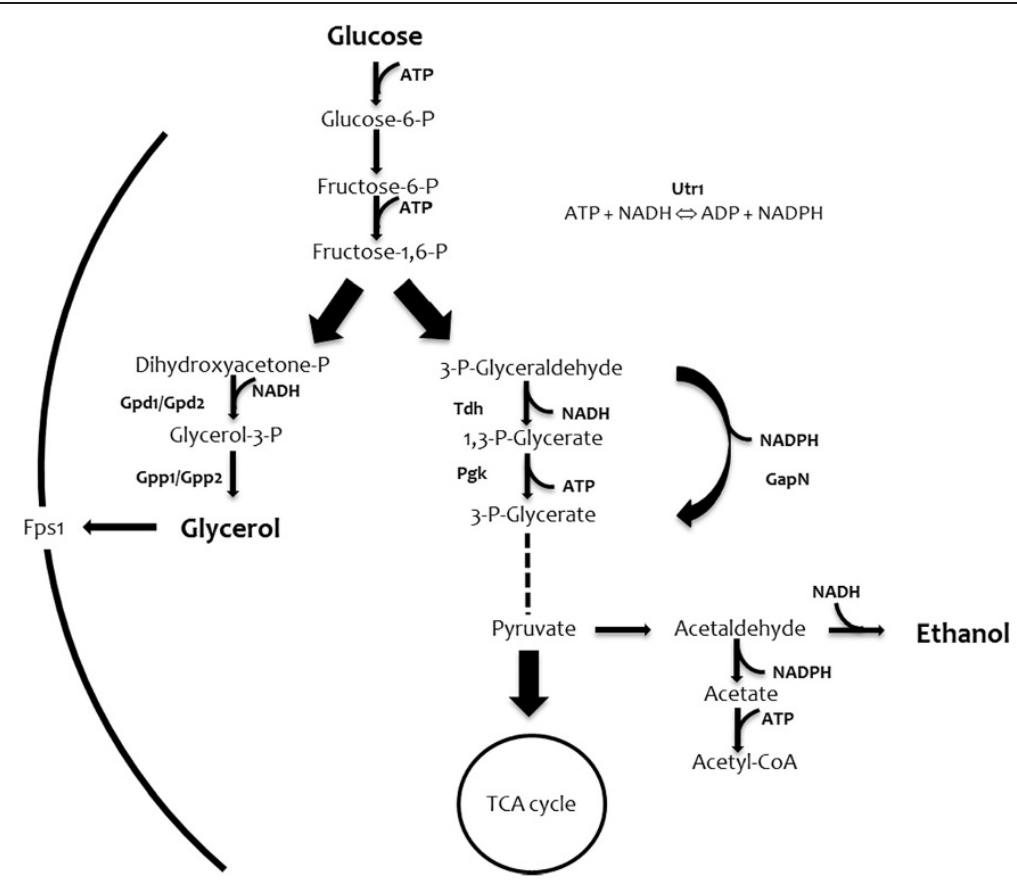

Figure 1 Schematic diagram of ethanol and glycerol metabolism in S. cerevisiae. Gpd1/Gpd2, glycerol 3-phosphate dehydrogenases; Gpp1/ Gpp2, glycerol 3-phosphate phosphatases; Tdh, glyceraldehyde 3-phosphate dehydrogenase; Pgk, phosphoglycerate kinase; Fps1, glycerol exporter (protein channel); TCA, tricarboxylic acid cycle. The strategy used in this work consists of heterologous expression of gapN (encoding $\mathrm{NADP}^{+}$-dependent glyceraldehyde 3-phosphate dehydrogenase) from S. mutans and overexpression of UTR1 (encoding ATP-NADH kinase) from S. cerevisiae.

2000a), but the double gpd1 1 gpd2 $\Delta$ mutant had a dramatically reduced specific growth rate under aerobic conditions with growth being completely abolished at anaerobic conditions (Bjorkqvist et al. 1997). To improve the ethanol yield while reducing glycerol formation, different approaches have been reported. To show whether a reduced formation of surplus NADH and an increased consumption of ATP in biosynthesis would result in a decreased glycerol yield and an increased ethanol yield in anaerobic cultivations, a yeast strain was constructed in which GLN1 (glutamine synthetase) and GLT1 (glutamate synthase) were overexpressed, and GDH1 $\left(\mathrm{NADP}^{+}\right.$-dependent glutamate dehydrogenase) was deleted (Nissen et al. 2000 b), which resulted in a $38 \%$ reduced glycerol yield. A genome-scale reconstructed metabolic network of $S$. cerevisiae was used to score the best strategies for metabolic engineering of the redox metabolism that would lead to decreased glycerol and increased ethanol yields, and this showed that expressing a non-phosphorylating, NADP ${ }^{+}$-dependent glyceraldehyde-3-phosphate dehydrogenase (GapN) was one of the best strategies tested (Bro et al. 2006). This has been confirmed in several studies, and it has also been shown that expression of GapN can rescue the negative effects from deletion of the glycerol export system Fps1 (Bro et al. 2006; Guo et al. 2009; Wang et al. 2011; Zhang et al. 2011). GapN catalyses the irreversible conversion of glyceraldehyde-3-phosphate and $\mathrm{NADP}^{+}$ into 3-phosphoglycerate and NADPH in glycolysis (Figure 1). With this strategy, production of glycerol is substituted with production of ethanol involving a net oxidation of NADH (Bro et al. 2006; Arnon et al. 1954). In another strategy to reduce glycerol production the Escherichia coli mhpF gene, encoding an acetylating NAD-dependent acetaldehyde dehydrogenase, was expressed in a gpd1 $1 \Delta p d 2 \Delta$ strain, and it was shown that anaerobic growth could be restored by supplementation with $2 \mathrm{~g} / \mathrm{l}$ acetic acid accompanied by reduced glycerol production (Guadalupe Medina et al. 2010).

Although it has been also shown that glycerol can cross the plasma membrane through a $\mathrm{H}^{+}$symport, detected in cells grown on non-fermentable carbon sources, and by passive diffusion (Gancedo et al. 1968; Lages and Lucas 1997; Lages et al. 1999; Oliveira et al. 2003), glycerol is mainly exported across the plasma membrane through the protein channel Fps1 (Remize et al. 2001) regulated by extracellular osmolarity (Tamas et al. 1999). Fps1 is a member of the major intrinsic protein (MIP) family of channel proteins. MIP channels have been reported to contain six putative transmembrane domains (Walz et al. 1997; Li et al. 1997; Stamer et al. 1996). Although Fps1 can transport glycerol in both directions, the main role of this glycerol facilitator is to regulate glycerol export rather than its uptake (Luyten et al. 1995; Tamas et al. 1999). The stress-activated protein kinase Hog1 can phosphorylate 
Fps1 triggering its endocytosis and further degradation under different stress conditions such as high acetic acid levels (Mollapour and Piper 2007). Fps1 is required for glycerol export under anaerobic conditions, and mutants lacking this protein grow poorly under anaerobiosis (Tamas et al. 1999). In an fps $1 \Delta$ mutant, intracellular glycerol accumulates since it cannot be exported. As a result, the accumulation of glycerol inside the yeast cells may induce other regulatory systems in order to reduce glycerol biosynthesis, thus resulting in an increase of the ethanol yield (Zhang et al. 2007).

$\mathrm{NAD}(\mathrm{H})$ kinases catalyse $\mathrm{NAD}(\mathrm{H})$ phosphorylation by using ATP or inorganic polyphosphate, constituting the last step of the $\operatorname{NADP}(\mathrm{H})$ biosynthetic pathway (Kawai et al. 2001; McGuinness and Butler 1985). S. cerevisiae is endowed with three $\mathrm{NAD}(\mathrm{H})$ kinase homologues, namely Utr1, Pos5 and Yel041w (Kawai et al. 2001; Outten and Culotta 2003; Strand et al. 2003). The ATP-NAD kinase Utr1 was proposed to participate in the ferrireductase system by supplying NADP (Kawai et al. 2001; Lesuisse et al. 1996). Pos5 was described as an ATP-NADH kinase, located in the mitochondrial matrix and reported to play an important role in several mitochondrial processes requiring NADPH. Cells lacking Pos5 accumulate mutations in the mitochondrial DNA and show poor growth in the presence of glycerol, oxidative damage and when growing in medium without arginine (Strand et al. 2003; Outten and Culotta 2003). Later on, Yel041w (re-named as Yef1) and Utr1 were identified as ATP-NADH kinases (Shi et al. 2005). Analysis of the single, double and triple mutants, which were found viable, showed the important contribution of Pos5 to the mitochondrial function and survival at high temperature $\left(37^{\circ} \mathrm{C}\right)$. The contribution of Utr1 to growth in low iron medium was also reported to be critical (Shi et al. 2005).

Here, we explored the combinatorial effect of reducing glycerol export and formation through deletion of FPS1, while expressing gapN from S. mutans and overexpressing the ATP-NADH kinase gene UTR1 from S. cerevisiae. With this strategy we aimed to solve the resulting redox balance problem and, at the same time, to increase the ethanol yield. We also analysed the effect on glycerol reduction/ethanol production, by using a set of different plasmids for varying expression levels of gapN and UTR1.

\section{Materials and methods}

\section{Strain construction and media}

The $S$. cerevisiae strains and plasmids used in this study are described in Table 1. CN1, which was used as the reference strain and CN6, where FPS1 was knocked-out, were transformed with empty plasmids. The FPS1 gene was deleted in $\mathrm{CN} 2$ to $\mathrm{CN} 6$ strains. $\mathrm{CN} 2$ to $\mathrm{CN} 5$ were transformed with different combinations of plasmids carrying gapN and UTR1 under control of the TEF1 promoter, and expressing the mentioned genes in high or low copy number (Table 1). Agar plates of synthetic medium (6.7 g/l yeast nitrogen base w/o aminoacids, $0.75 \mathrm{~g} / \mathrm{l}$ complete supplement mixture w/o uracil, $2 \%$ glucose, $2 \%$ agar, $\mathrm{pH} 6$ ) or YPD ( $2 \%$ glucose, $2 \%$ peptone, $1 \%$ yeast extract, $2 \%$ agar) supplemented with $200 \mathrm{mg} / \mathrm{l}$ of G418, were used for selection of the strains. For the fermentations, yeast cells were grown in minimal synthetic

Table $1 \mathrm{~S}$. cerevisiae strains and plasmids used in this work

\begin{tabular}{|c|c|c|}
\hline Strains & Genotype description & Reference \\
\hline CEN.PK 113-11C & MATa his341 ura3-52 MAL2-8 SUC2 & (van Dijken et al. 2000) \\
\hline CEN.PK 113-11C; p426; p423 (CN1) & MATa his341 ura3-52 MAL2-8 S SUC2 p426TEF1 p423TEF1 & This work \\
\hline fps1D; p426-GapN; p423-UTR1 (CN2) & MATa his341 ura3-52 MAL2-8c SUC2 fps1D p426TEF1-GapN p423TEF1-UTR1 & This work \\
\hline fps14; p426-GapN; p413-UTR1 (CN3) & MATa his341 ura3-52 MAL2-8 SUC2 fps1D p426TEF1-GapN p413TEF1-UTR1 & This work \\
\hline fps14; p416-GapN; p423-UTR1 (CN4) & MATa his341 ura3-52 MAL2-8 SUC2 fps1D p416TEF1-GapN p423TEF1-UTR1 & This work \\
\hline fps14; p416-GapN; p413-UTR1 (CN5) & MATa his341 ura3-52 MAL2-8 SUC2 fps1D p416TEF1-GapN p413TEF1-UTR1 & This work \\
\hline fps1 $;$; p426; p423 (CN6) & MATa his341 ura3-52 MAL2-8 SUC2 fps1 $\triangle$ p426TEF1 p423TEF1 & This work \\
\hline \multicolumn{3}{|l|}{ Plasmids } \\
\hline pCIChE-KK004-Gap & neor, integrative plasmid & (Kocharin 2013) \\
\hline p426TEF1 & URA3, $2 \mu$ plasmid, TEF1 promoter, CYC1 terminator & (Mumberg et al. 1995) \\
\hline p416TEF1 & URA3, centromeric plasmid, TEF1 promoter, CYC1 terminator & (Mumberg et al. 1995) \\
\hline p423TEF1 & HIS3, 2 M plasmid, TEF1 promoter, CYC1 terminator & (Mumberg et al. 1995) \\
\hline p413TEF1 & HIS3, centromeric plasmid, TEF1 promoter, CYC1 terminator & (Mumberg et al. 1995) \\
\hline p426TEF1-GapN & URA3, $2 \mu$ plasmid, gapN from S. mutans & This work \\
\hline p416TEF1-GapN & URA3, centromeric plasmid, gapN from S. mutans & This work \\
\hline p423TEF1-UTR1 & HIS3, 2 u plasmid, UTR1 & This work \\
\hline p413TEF1-UTR1 & HIS3, centromeric plasmid, UTR1 & This work \\
\hline
\end{tabular}


medium (Verduyn et al. 1992) containing $20 \mathrm{~g} / \mathrm{l}$ glucose. The $\mathrm{pH}$ was adjusted to 5.2 with $2 \mathrm{M} \mathrm{KOH}$ before sterilization. In bioreactors, $10 \mathrm{mg} / \mathrm{l}$ of ergosterol and $420 \mathrm{mg} / \mathrm{l}$ of Tween 80 were added to the medium for the anaerobic growth of $S$. cerevisiae.

\section{Deletion of FPS1}

The FPS1 gene was replaced, by using the loxP-kanMXloxP cassette obtained by PCR from the plasmid pUG6 (Wach et al. 1994) and a bipartite strategy (Erdeniz et al. 1997). Primers containing upstream and downstream homologous regions of FPS1 were used for that purpose (Table 2). The PCR products were used for transformation of CEN.PK 113-11C, and the transformed cells were selected in G418-YPD medium. The selectable marker was removed afterwards by expression of the Cre recombinase from plasmid pSH47 under the control of a galactose inducible promoter (Guldener et al. 1996). The correct integration of the fragment disrupting the coding sequence was further tested by PCR and sequencing.

\section{Heterologous expression of gapN and overexpression of UTR1}

The sequence of the $\mathrm{NADP}^{+}$-dependent glyceraldehyde 3-phosphate dehydrogenase gene gapN from S. mutans (Gene ID: 1028095) was obtained from plasmid pCIChEKK004-Gap and cloned into p426TEF1 and p416TEF1, respectively, by using PCR and restriction enzymes (REs). BamHI and ClaI sites were additionally included in the designed PCR-primers for that purpose (Table 2). The UTR1 sequence (Gene ID: 853508) was obtained by PCR from CEN.PK 113-11C genomic DNA and cloned into p423TEF1 and p413TEF1 plasmids by REs. In this case, the BamHI/EcoRI combination was used (Table 2). The constructs were checked by PCR for gapN or UTR1 genes, digestion with the proper RE combination and sequencing.

Batch cultivations in flasks and analytical methods

Seed cultures of yeast cells were grown during 20-24 hours in shake flasks containing $25 \mathrm{ml}$ of minimal medium at $30^{\circ} \mathrm{C}$ and constant shaking (120 rpm). Micro-aerobic conditions were obtained by using $150 \mathrm{ml}$ flasks covered with fermentation bungs and containing $100 \mathrm{ml}$ of medium (Arnon et al. 1954). The shake flasks were inoculated with an initial $\mathrm{OD}_{600}$ of 0.05 and grown at $30^{\circ} \mathrm{C}(120-140 \mathrm{rpm})$. Samples of $2 \mathrm{ml}$ of culture were taken at the selected timepoints for optical density and HPLC measurements. Supernatants centrifuged for $5 \mathrm{~min}$ at high speed $(1 \mathrm{ml})$ were loaded to a HPX-87G column (Biorad, Hercules, CA) on a Dionex Ultimate 3000 HPLC (Dionex Softron GmbH, Jülich, Germany) to measure the concentrations of glucose, ethanol, glycerol and acetate in the cultures at the different time points. The samples were run at a flow rate of $0.6 \mathrm{ml} /$ min at $65^{\circ} \mathrm{C}$, using $5 \mathrm{mM} \mathrm{H}_{2} \mathrm{SO}_{4}$ as mobile phase. Fermentation experiments were repeated three independent times.

\section{Fermentation analysis in bioreactors}

Yeast cell seed cultures were grown in $25 \mathrm{ml}$ of minimal medium supplemented with $20 \mathrm{~g} / \mathrm{l}$ of glucose, and incubated at $30^{\circ} \mathrm{C}$ for $22-24$ hours $(130 \mathrm{rpm})$. The experiment was performed in $1 \mathrm{l}$ stirrer-pro vessels (DasGip $\mathrm{GmbH}$, Jülich, Germany) with a working volume of $0.5 \mathrm{l}$, and bioreactors were inoculated at initial $\mathrm{OD}_{600}$ of 0.02 . The cells were centrifuged and washed with $5 \mathrm{ml}$ of water before inoculation.

The temperature was controlled at $30^{\circ} \mathrm{C}$ using a DasGip Bioblock integrated heating and cooling thermo well. Agitation was maintained at $600 \mathrm{rpm}$ using an overhead drive stirrer with one Rushton impeller. The bioreactors were flushed with $\mathrm{N}_{2}$ at $1 \mathrm{vvm}$ when anaerobic growth conditions were required. The $\mathrm{pH}$ was maintained constant at 5.0 by the automatic addition of $2 \mathrm{M} \mathrm{KOH}$.

\section{Results}

Yeast cells expressing the gapN/UTR1 genes show an increased ethanol production capacity and reduced glycerol production.

Strains deleted in FPS1 and expressing gapN and UTR1 from either high or low copy number plasmids were cultivated in shake flasks under micro-aerobic conditions.

Table 2 Oligonucleotide primers used in this study

\begin{tabular}{|c|c|c|}
\hline Primer name & Primer sequence $\left(5^{\prime}-3^{\prime}\right)$ & Restriction sites ${ }^{*}$ \\
\hline kanMX_FPS1_F & TATTTTCGATCAGATCTCATAGTGAGAAGGCGCAATTCAGTAGTTGGCATCAGAGCAGATTGTACTGAGAG & \\
\hline kanMX_R & AAACTCACCGAGGCAGTTCCATAG & \\
\hline kanMX_F & ATGGTCAGACTAAACTGGCTGACG & \\
\hline kanMX_FPS1_R & САTCCATGCGATACATCATGTATAGTAGGTGACCAGGCTGAGTTCTACCGCCTTGAGTGAGCTGATAC & \\
\hline UTR1_F & CGGGATCCCGAAAACAATGAAGGAGAATGACATGAATA & $\mathrm{BamHI}$ \\
\hline UTR1_R & CGGAATTCCGGTAACATTATACTGAAAACCTTGCTTGA & EcoRI \\
\hline GapN_F & CGGGATCCCGAAAACAATGACAAAACAATACAAAAACT & $\mathrm{BamHI}$ \\
\hline GapN_R & CCATCGATGGAGATCTTCACTITATGTCAAAGACAACA & Clal \\
\hline
\end{tabular}


Glucose consumption was completed in CN1 (reference strain) after around $30 \mathrm{~h}$ of fermentation. For the rest of strains, this level was reached first after about $37 \mathrm{~h}$. The CN6 strain (FPS1 deletion strain carrying both empty plasmids) showed $0.5-1 \mathrm{~g} / \mathrm{l}$ of remaining glucose still after 45 hours of fermentation and the final ethanol production, for this strain, was much lower in comparison to the rest of the transformants tested (Figure 2). This correlates with the specific growth rate of the analysed strains (Table 3).

In the case of the reference strain, the highest level of ethanol was reached already after $25 \mathrm{~h}$ of fermentation, whereas for the rest of the strains we could observe a more gradual production over time until reaching the highest ethanol production (Figure 2). Ethanol production after 35-40 h of fermentation was improved in strains $\mathrm{CN} 2$ to $\mathrm{CN} 5$ expressing the alternative enzymes to reduce NADH levels (Figure 2). These results were confirmed when the specific ethanol productivity was calculated for these strains (Table 3). Strain CN6 grew very poorly hence affecting the calculation of ethanol productivity, and data for this strain were therefore not included in the further analysis.

Glycerol production was reduced in strains CN2 to CN6, confirming our initial hypothesis, showing only a minor effect on cell growth. An exception is strain CN6, which suffers from the lack of an NADH sink and hence has a much reduced growth rate (Figure 2 and Table 3).

Fermentation analysis in bioreactors showed that, except for the reference strain (CN1), cells were not able to grow under strict anaerobic conditions. Adaptation was necessary in order to complete the fermentation profile of the strains. This problem was partially solved by sparging air containing $12 \%$ oxygen at the beginning of fermentation. Under these conditions cells were able to grow, consume the carbon source and produce ethanol.

The ATP consumption by Utr1 and the inhibition of ATP formation by GapN, could be one of the reasons why the engineered strains showed a reduction in their ability to grow anaerobically.

Analytical methods revealed that results from individual experiments were similar to those obtained in shake flasks, with a higher ethanol production and glycerol reduction in the engineered strains (CN2-CN5) compared to the reference strain. But as a consequence of the previous adaptation needed, results were not very consistent between experiments and cells did not always grow at the same level, making it very difficult to represent the data as average yields of the different fermentation experiments. We concluded that more investigations are needed when growing cells in bioreactors, in order to use this strategy for industrial purposes, and therefore,
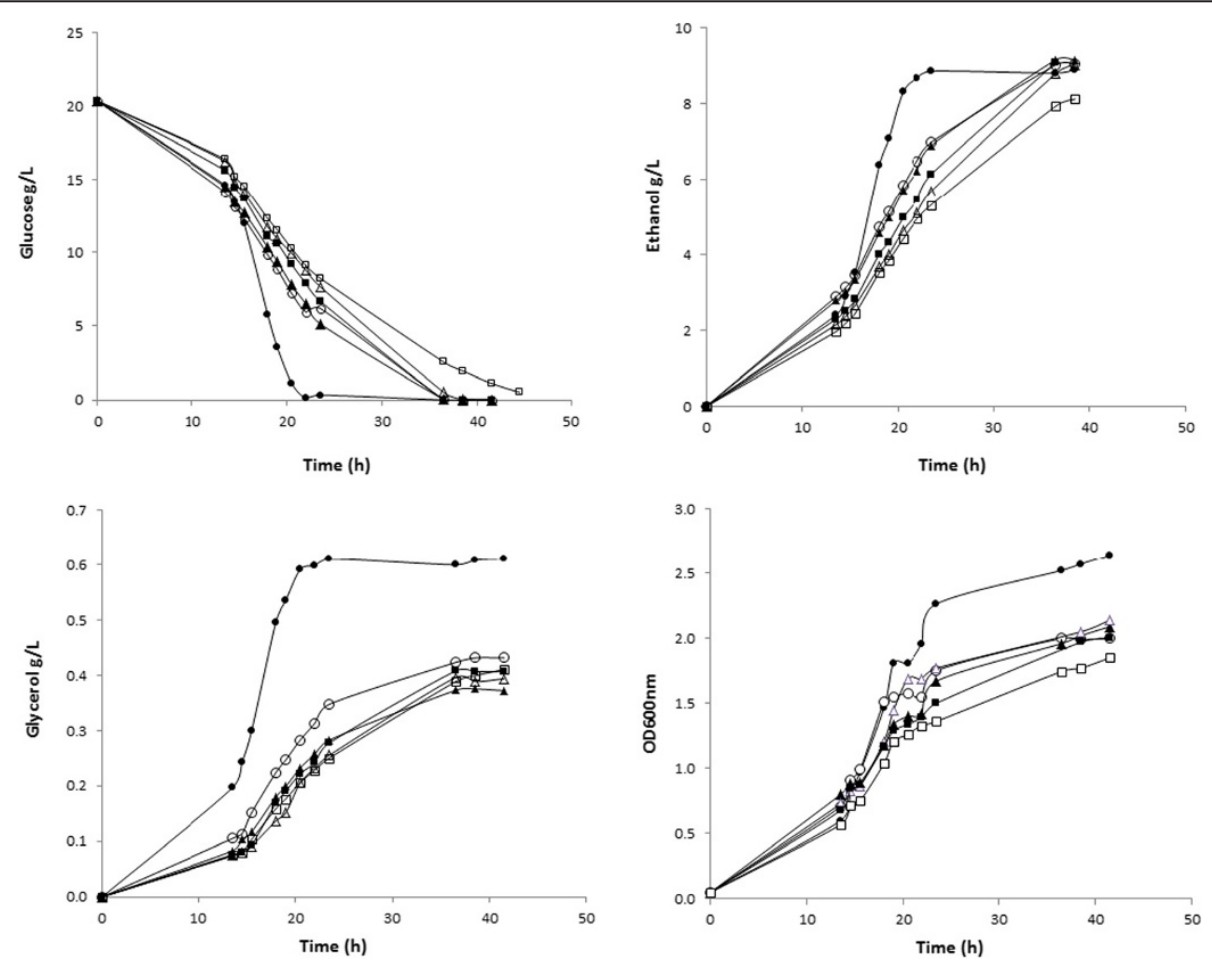

Figure 2 Growth curves, glucose, ethanol and glycerol profiles during micro-aerobic conditions of Saccharomyces cerevisiae. (•) CN1,

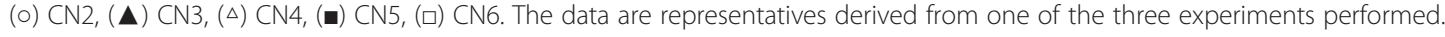


Table 3 Compound yields, growth and specific ethanol productivity in engineered yeast strains

\begin{tabular}{|c|c|c|c|c|c|c|}
\hline Strain & Ethanol yield $(g / g)^{*}$ & Ethanol increase (\%) & Glycerol yield $(\mathbf{g} / \mathrm{g})^{*}$ & Acetate yield $(g / g)^{*}$ & $\mu\left(h^{-1}\right)$ & $q_{s p}\left(g^{-1} h^{-1}\right)$ \\
\hline $\begin{array}{l}\text { CEN.PK 113-11C; } \\
\text { p426; p423 (CN1) }\end{array}$ & $0.449 \pm 0.011$ & - & $0.042 \pm 0.018$ & $0.0033 \pm 0.00028$ & $0.18 \pm 0.048$ & $26.55 \pm 0.293$ \\
\hline $\begin{array}{l}\text { fps 14; p426-GapN; } \\
\text { p423-UTR1 (CN2) }\end{array}$ & $0.466 \pm 0.013$ & 3.78 & $0.030 \pm 0.012$ & $0.0038 \pm 0.00014$ & $0.11 \pm 0.059$ & $51.71 \pm 0.440$ \\
\hline $\begin{array}{l}\text { fps14; p426-GapN; } \\
\text { p413-UTR1 (CN3) }\end{array}$ & $0.470 \pm 0.018$ & 4.67 & $0.026 \pm 0.010$ & $0.0039 \pm 0.00085$ & $0.11 \pm 0.052$ & $52.02 \pm 0.648$ \\
\hline $\begin{array}{l}\text { fps14; p416-GapN; } \\
\text { p423-UTR1 (CN4) }\end{array}$ & $0.457 \pm 0.014$ & 1.78 & $0.027 \pm 0.012$ & $0.0046 \pm 0.00134$ & $0.13 \pm 0.046$ & $40.47 \pm 0.609$ \\
\hline $\begin{array}{l}\text { fps14; p416-GapN; } \\
\text { p413-UTR1 (CN5) }\end{array}$ & $0.458 \pm 0.012$ & 2 & $0.029 \pm 0.013$ & $0.0032 \pm 0.00021$ & $0.11 \pm 0.038$ & $52.37 \pm 1.208$ \\
\hline fps14; p426; p423 (CN6) & $0.443 \pm 0.0040$ & -1.33 & $0.023 \pm 0.00049$ & $0.0096 \pm 0.00021$ & $0.070 \pm 0.0011$ & - \\
\hline
\end{tabular}

Data represent the means of results from triplicates \pm SD.

${ }^{*} \mathrm{~g} / \mathrm{g}$ is equivalent to gram of produced compound per gram of glucose. $\mu$ represents the specific growth rate.

$\mathrm{q}_{\mathrm{sp}}$ represents the specific ethanol productivity.

we focused on the results we obtained in micro-aerobic conditions to analyse our strategy.

\section{Physiological characterization of the engineered yeast strains. Effect of the gene copy number of gapN and UTR1 genes on ethanol yield}

At the end of fermentation process, the ethanol yield was improved in the transformant strains by $1.7-4.6 \%$ (depending on the strain analysed) compared to the reference strain $(\mathrm{CN} 1)$. $\mathrm{CN} 2$ and $\mathrm{CN} 3$ were the best ethanol producers ( $\mathrm{p}$-value $<0.05$ after Student's t-test), showing ethanol yields up to 0.47 grams per gram of glucose (Table 3). After the analysis of the possible effect of high/low expression of gapN and UTR1, by using different expression plasmids, we could observe the improvement in $\mathrm{CN} 2$ and $\mathrm{CN} 3$ strains both carrying gapN on a high-copy plasmid. In the case of UTR1, the copy number of the kinase gene did not seem to be crucial for a better ethanol yield, since the best producer (CN3) expressed UTR1 from a low-copy plasmid. These results suggest a more important role of the $\mathrm{NADP}^{+}$-dependent glyceraldehyde 3-phosphate dehydrogenase in the redox balancing for improved ethanol production.

To further check this hypothesis, we performed an analysis of the fps1D strain expressing gapN or UTR1 individually (both from a high-copy plasmid). The fps $1 \Delta$ strain expressing gapN showed similar growth and final ethanol production compared to $\mathrm{CN} 2$ and $\mathrm{CN} 3$ strains, (7.98 g/l versus 8.14 and $8.43 \mathrm{~g} / \mathrm{l}$ respectively) after $\sim 40 \mathrm{~h}$ of fermentation in $20 \mathrm{~g} / \mathrm{l}$ of glucose. On the other hand, the fps $1 \Delta$ strain expressing UTR1 grew similar to the CN6 control strain. Ethanol production at the end of fermentation was also at the same level as measured in the CN6 strain $(5.6 \mathrm{~g} / \mathrm{l}$ versus $6.7 \mathrm{~g} / \mathrm{l})$.

Furthermore, for the transformant strains the glycerol yield was successfully reduced by $25-40 \%$ compared to the reference strain. Although the transformant strains
CN2-CN5 showed a specific growth rate reduced by 27-38\%, they could still grow at a good level and entirely consume the carbon source. As explained before, the CN6 strain with highest glycerol reduction was the one most affected in terms of specific growth rate and also regarding ethanol production (Table 3 and Figure 2).

\section{Discussion}

In $S$. cerevisiae, it is well known from previously published studies that, under aerobic conditions, fps $1 \Delta$ mutants show a growth profile similar to a wild type, due to NADH re-oxidation by the respiratory chain (Zhang et al. 2007). Under anaerobic conditions, glycerol production is the main redox-sink for the excess of NADH (Valadi et al. 2004). When glycerol export is blocked, the fps $1 \Delta$ mutants produce less glycerol and biomass, and show a lower glucose uptake rate compared to the reference strain. However, in case of the mutant strain, there is residual glucose in the growth medium, resulting in a decrease of total ethanol produced (Wang et al. 2011). As already described in the results, the final ethanol production for the CN6 strain was much lower in comparison to the rest of the transformants tested (Figure 2). Moreover, it has been previously described that acetic acid enters glucose-repressed yeast cells primarily by facilitated diffusion through Fps1. The total loss of the channel also creates an acetate resistant phenotype by elimination of the major source of the acetic acid flux into the cell (Mollapour and Piper 2007). The increased acetate yield observed in the CN6 strain might be connected to an inhibited acetate import in these cells (Table 3).

GapN catalyses the irreversible conversion of glyceraldehyde-3-phosphate and $\mathrm{NADP}^{+}$into 3-phosphoglycerate and NADPH in glycolysis, and in contrast to the native yeast pathway, no NADH and ATP are released in this reaction (Figure 1). Expression of gapN has a high potential 
to reduce NADH generation, and may therefore lead to a complete redirection of flux from formation of glycerol to ethanol (Bro et al. 2006). When alternative routes for NADH re-oxidation were expressed, ethanol production was improved in strains CN2 to CN5 (Figure 2).

The intracellular concentration of the $\mathrm{NADP}^{+} / \mathrm{NADPH}$ pool is much lower than that of the $\mathrm{NAD}^{+} / \mathrm{NADH}$ pool, and shifting part of the glycolytic flux from using $\mathrm{NAD}^{+}$ to using $\mathrm{NADP}^{+}$as co-factor may therefore result in limitation of supply of this co-factor. We therefore evaluated whether increasing the $\mathrm{NADP}^{+} / \mathrm{NADPH}$ pool by overexpressing the UTR1 gene encoding an NADH-kinase may have a positive effect on top of the use of GapN as a glycolytic enzyme. As already mentioned and represented in Figure 1, NAD $(H)$ kinases catalyse $\operatorname{NAD}(H)$ phosphorylation by using ATP or inorganic polyphosphate in yeast, constituting the last step of the NADP biosynthetic pathway (McGuinness and Butler 1985; Kawai et al. 2001). UTR1 overexpression does, however, not seem to lead to an effect on ethanol and glycerol production.

Regarding glycerol production, it is believed that the complete elimination of its production is not practical due to its importance in osmoregulation and maintenance of the intracellular redox balance (Blomberg and Adler 1992; van Dijken and Scheffers 1986). Moreover, it is a precursor used to synthesize the cellular membrane. On the other hand, it can be reduced to a minimal level in order to improve the ethanol yield (Bjorkqvist et al. 1997; Nissen et al. 2000b). Our hypothesis was confirmed and glycerol production was reduced in the strains CN2-CN6, Except for strain CN6, which suffers from the lack of an NADH sink, the cell growth was only mildly affected (Figure 2 and Table 3 ).

The present work evidences that the function of glycerol as a redox sink for anaerobic growth can be successfully replaced by introducing a heterologous GapN pathway from $S$. mutans in addition to deletion of the FPS1 gene (responsible for glycerol export) and overexpression of the NADH-kinase Utr1, resulting in a reduction of glycerol (by-product) formation together with an increase in the ethanol yield.

By expressing gapN (in combination with the overexpression of UTR1) we can solve the redox problem in the fps $1 \Delta$ mutant thereby increasing the specific growth rate and reducing the formation of NADH. Thus, the carbon source was redirected towards ethanol production in the engineered strains, which increased the ethanol yield by up to $4.6 \%$ compared to the reference strain.

\section{Competing interests}

The authors declare that they have no competing interest.

\section{Authors' contributions}

CN, JN and VS participated in the design of the experiment. CN performed all the experiments, analyzed the data and wrote the manuscript. JN and VS edited the manuscript. All authors read and approved the final manuscript.

\section{Acknowledgements}

The authors would like to thank The Swedish Research Council (Vetenskapsrådet) and Novozymes A/S for financial support. Francesco Gatto is acknowledged for his help with the statistical analysis of the data. We also thank Michael L. Nielsen, Pia Francke Johannesen and Carsten Hjort for helpful suggestions and comments about this work.

Received: 1 October 2014 Accepted: 27 November 2014

Published online: 11 December 2014

\section{References}

Andre L, Hemming A, Adler L (1991) Osmoregulation in Saccharomyces cerevisiae. Studies on the osmotic induction of glycerol production and glycerol-3phosphate dehydrogenase (NAD+). FEBS Lett 286(1-2):13-17

Ansell R, Granath K, Hohmann S, Thevelein JM, Adler L (1997) The two isoenzymes for yeast NAD + -dependent glycerol 3-phosphate dehydrogenase encoded by GPD1 and GPD2 have distinct roles in osmoadaptation and redox regulation. EMBO J 16(9):2179-2187, doi:10.1093/emboj/16.9.2179

Arnon DI, Rosenberg LL, Whatley FR (1954) A New Glyceraldehyde Phosphate Dehydrogenase from Photosynthetic Tissues. Nature 173(4415):1132-1134

Bjorkqvist S, Ansell R, Adler L, Liden G (1997) Physiological response to anaerobicity of glycerol-3-phosphate dehydrogenase mutants of Saccharomyces cerevisiae. Appl Environ Microbiol 63(1):128-132

Blomberg A, Adler L (1992) Physiology of osmotolerance in fungi. Adv Microb Physiol 33:145-212

Bro C, Regenberg B, Forster J, Nielsen J (2006) In silico aided metabolic engineering of Saccharomyces cerevisiae for improved bioethanol production. Metab Eng 8(2):102-111, doi:10.1016/j.ymben.2005.09.007

Erdeniz N, Mortensen UH, Rothstein R (1997) Cloning-free PCR-based allele replacement methods. Genome Res 7(12):1174-1183

Eriksson P, Andre L, Ansell R, Blomberg A, Adler L (1995) Cloning and characterization of GPD2, a second gene encoding sn-glycerol 3-phosphate dehydrogenase (NAD+) in Saccharomyces cerevisiae, and its comparison with GPD1. Mol Microbiol 17(1):95-107

Gancedo C, Gancedo JM, Sols A (1968) Glycerol Metabolism in Yeasts - Pathways of Utilization and Production. Eur J Biochem 5(2):165, doi:10.1111/j.1432 1033.1968.tb00353.x

Guadalupe Medina V, Almering MJ, van Maris AJ, Pronk JT (2010) Elimination of glycerol production in anaerobic cultures of a Saccharomyces cerevisiae strain engineered to use acetic acid as an electron acceptor. Appl Environ Microbiol 76(1):190-195, doi:10.1128/AEM.01772-09

Guldener U, Heck S, Fielder T, Beinhauer J, Hegemann JH (1996) A new efficient gene disruption cassette for repeated use in budding yeast. Nucleic Acids Res 24(13):2519-2524

Guo ZP, Zhang L, Ding ZY, Wang ZX, Shi GY (2009) Interruption of glycerol pathway in industrial alcoholic yeasts to improve the ethanol production. Appl Microbiol Biotechnol 82(2):287-292, doi:10.1007/s00253-008-1777-7

Hahn-Hagerdal B, Karhumaa K, Fonseca C, Spencer-Martins I, Gorwa-Grauslund MF (2007) Towards industrial pentose-fermenting yeast strains. Appl Microbiol Biotechnol 74(5):937-953, doi:10.1007/s00253-006-0827-2

Kawai S, Suzuki S, Mori S, Murata K (2001) Molecular cloning and identification of UTR1 of a yeast Saccharomyces cerevisiae as a gene encoding an NAD kinase. FEMS Microbiol Lett 200(2):181-184

Kocharin K (2013) Metabolic engineering of Saccharomyces cerevisiae for polyhydroxybutyrate production. In: Thesis. Chalmers University of Technology, Sweden

Lages F, Lucas C (1997) Contribution to the physiological characterization of glycerol active uptake in Saccharomyces cerevisiae. Bba-Bioenergetics 1322(1):8-18, doi:10.1016/S0005-2728(97)00062-5

Lages F, Silva-Graca M, Lucas C (1999) Active glycerol uptake is a mechanism underlying halotolerance in yeasts: a study of 42 species. Microbiol-Uk 145:2577-2585

Larsson K, Ansell R, Eriksson P, Adler L (1993) A gene encoding sn-glycerol 3-phosphate dehydrogenase (NAD+) complements an osmosensitive mutant of Saccharomyces cerevisiae. Mol Microbiol 10(5):1101-1111

Lesuisse E, Casteras-Simon M, Labbe P (1996) Evidence for the Saccharomyces cerevisiae ferrireductase system being a multicomponent electron transport chain. J Biological Chem 271(23):13578-13583

Li H, Lee S, Jap BK (1997) Molecular design of aquaporin-1 water channel as revealed by electron crystallography. Nat Struct Mol Biol 4(4):263-265 
Luyten K, Albertyn J, Skibbe WF, Prior BA, Ramos J, Thevelein JM, Hohmann S (1995) Fps1, a yeast member of the MIP family of channel proteins, is a facilitator for glycerol uptake and efflux and is inactive under osmotic stress. EMBO J 14(7):1360-1371

McGuinness ET, Butler JR (1985) NAD+ kinase-a review. Int J Biochem 17(1):1-11

Michnick S, Roustan JL, Remize F, Barre P, Dequin S (1997) Modulation of glycerol and ethanol yields during alcoholic fermentation in Saccharomyces cerevisiae strains overexpressed or disrupted for GPD1 encoding glycerol 3-phosphate dehydrogenase. Yeast 13(9):783-793, doi:10.1002/(SICI)1097-0061(199707) 13:9 < 783::AID-YEA128> 3.0.CO;2-W

Mollapour M, Piper PW (2007) Hog1 mitogen-activated protein kinase phosphorylation targets the yeast Fps1 aquaglyceroporin for endocytosis, thereby rendering cells resistant to acetic acid. Mol Cell Biol 27(18):6446-6456, doi:10.1128/MCB.02205-06

Mumberg D, Muller R, Funk M (1995) Yeast vectors for the controlled expression of heterologous proteins in different genetic backgrounds. Gene 156(1):119-122

Nissen TL, Hamann CW, Kielland-Brandt MC, Nielsen J, Villadsen J (2000a) Anaerobic and aerobic batch cultivations of Saccharomyces cerevisiae mutants impaired in glycerol synthesis. Yeast 16(5):463-474, doi:10.1002/(SICI) 1097-0061(20000330)16:5 < 463::AID-YEA535 > 3.0.CO;2-3

Nissen TL, Kielland-Brandt MC, Nielsen J, Villadsen J (2000b) Optimization of ethanol production in Saccharomyces cerevisiae by metabolic engineering of the ammonium assimilation. Metab Eng 2(1):69-77, doi:10.1006/mben.1999.0140

Oliveira R, Lages F, Silva-Graca M, Lucas C (2003) Fps1p channel is the mediator of the major part of glycerol passive diffusion in Saccharomyces cerevisiae: artefacts and re-definitions. Bba-Biomembranes 1613(1-2):57-71, doi:10.1016/S0005-2736(03)00138-X

Oura E (1977) Reaction products of yeast fermentations. Process Biochem 12(35):19-21

Outten CE, Culotta VC (2003) A novel NADH kinase is the mitochondrial source of NADPH in Saccharomyces cerevisiae. EMBO J 22(9):2015-2024, doi:10.1093/emboj/cdg211

Remize F, Barnavon L, Dequin S (2001) Glycerol export and glycerol-3-phosphate dehydrogenase, but not glycerol phosphatase, are rate limiting for glycerol production in Saccharomyces cerevisiae. Metab Eng 3(4):301-312, doi:10.1006/mben.2001.0197

Shi F, Kawai S, Mori S, Kono E, Murata K (2005) Identification of ATP-NADH kinase isozymes and their contribution to supply of NADP(H) in Saccharomyces cerevisiae. FEBS J 272(13):3337-3349, doi:10.1111/j.1742-4658.2005.04749.x

Stamer WD, Snyder RW, Regan JW (1996) Characterization of the transmembrane orientation of aquaporin-1 using antibodies to recombinant fusion proteins. Biochemistry 35(50):16313-16318, doi:10.1021/bi9619536

Strand MK, Stuart GR, Longley MJ, Graziewicz MA, Dominick OC, Copeland WC (2003) POS5 gene of Saccharomyces cerevisiae encodes a mitochondrial $\mathrm{NADH}$ kinase required for stability of mitochondrial DNA. Eukaryotic Cell 2(4):809-820

Tamas MJ, Luyten K, Sutherland FC, Hernandez A, Albertyn J, Valadi H, Li H, Prior BA, Kilian SG, Ramos J, Gustafsson L, Thevelein JM, Hohmann S (1999) Fps1p controls the accumulation and release of the compatible solute glycerol in yeast osmoregulation. Mol Microbiol 31(4):1087-1104

Valadi H, Valadi A, Ansell R, Gustafsson L, Adler L, Norbeck J, Blomberg A (2004) $\mathrm{NADH}$-reductive stress in Saccharomyces cerevisiae induces the expression of the minor isoform of glyceraldehyde-3-phosphate dehydrogenase (TDH1). Curr Genet 45(2):90-95, doi:10.1007/s00294-003-0469-1

van Dijken JP, Bauer J, Brambilla L, Duboc P, Francois JM, Gancedo C, Giuseppin ML, Heijnen JJ, Hoare M, Lange HC, Madden EA, Niederberger P, Nielsen J, Parrou JL, Petit T, Porro D, Reuss M, van Riel N, Rizzi M, Steensma HY, Verrips $C T$, Vindelov J, Pronk JT (2000) An interlaboratory comparison of physiological and genetic properties of four Saccharomyces cerevisiae strains. Enzym Microb Technol 26(9-10):706-714

van Dijken JP, Scheffers WA (1986) Redox balances in the metabolism of sugars by yeasts. FEMS Microbiol Lett 32(3-4):199-224, doi:10.1111/j.1574-6968.1986. tb01194.x

van Maris AJ, Abbott DA, Bellissimi E, van den Brink J, Kuyper M, Luttik MA, Wisselink HW, Scheffers WA, van Dijken JP, Pronk JT (2006) Alcoholic fermentation of carbon sources in biomass hydrolysates by Saccharomyces cerevisiae: current status. Antonie Van Leeuwenhoek 90(4):391-418, doi:10.1007/s10482-006-9085-7
Vemuri GN, Eiteman MA, McEwen JE, Olsson L, Nielsen J (2007) Increasing NADH oxidation reduces overflow metabolism in Saccharomyces cerevisiae. Proc Natl Acad Sci U S A 104(7):2402-2407, doi:10.1073/pnas.0607469104

Verduyn C, Postma E, Scheffers WA, Van Dijken JP (1992) Effect of benzoic acid on metabolic fluxes in yeasts: a continuous-culture study on the regulation of respiration and alcoholic fermentation. Yeast 8(7):501-517, doi:10.1002/ yea. 320080703

Wach A, Brachat A, Pohlmann R, Philippsen P (1994) New heterologous modules for classical or PCR-based gene disruptions in Saccharomyces cerevisiae. Yeast 10(13):1793-1808

Walz T, Hirai T, Murata K, Heymann JB, Mitsuoka K, Fujiyoshi Y, Smith BL, Agre P, Engel A (1997) The three-dimensional structure of aquaporin-1. Nature 387(6633):624-627, doi:10.1002/jctb.2634

Wang P-M, Zheng D-Q, Ding R, Chi X-Q, Tao X-L, Min H, Wu X-C (2011) Improvement of ethanol production in Saccharomyces cerevisiae by hetero-expression of GAPN and FPS1 deletion. J Chem Technol Biotechnol 86(9):1205-1210, doi:10.1002/jctb.2634

Wyman C, Hinman N (1990) Ethanol. Appl Biochem Biotechnol 24-25(1):735-753, doi:10.1007/BF02920291

Zhang A, Chen X (2008) Improve Ethanol Yield Through Minimizing Glycerol Yield in Ethanol Fermentation of Saccharomyces cerevisiae. Chin J Chem Eng 16(4):620-625, doi:http://dx.doi.org/10.1016/S1004-9541(08)60130-5

Zhang A, Kong Q, Cao L, Chen X (2007) Effect of FPS1 deletion on the fermentation properties of Saccharomyces cerevisiae. Lett Appl Microbiol 44(2):212-217, doi:10.1111/j.1472-765X.2006.02041.X

Zhang L, Tang Y, Guo ZP, Ding ZY, Shi GY (2011) Improving the ethanol yield by reducing glycerol formation using cofactor regulation in Saccharomyces cerevisiae. Biotechnol Lett 33(7):1375-1380, doi:10.1007/s10529-011-0588-6

\section{doi:10.1186/s13568-014-0086-z}

Cite this article as: Navarrete et al.: Enhanced ethanol production and reduced glycerol formation in fps $1 \Delta$ mutants of Saccharomyces cerevisiae engineered for improved redox balancing. $A M B$ Express 2014 4:86

\section{Submit your manuscript to a SpringerOpen ${ }^{\odot}$ journal and benefit from:}

- Convenient online submission

$\checkmark$ Rigorous peer review

- Immediate publication on acceptance

- Open access: articles freely available online

- High visibility within the field

- Retaining the copyright to your article

Submit your next manuscript at $>$ springeropen.com 